\title{
The Impact of the Recurrent Larynge
Injury on Prognosis After McKeown Esophagectomy for ESCC
}

This article was published in the following Dove Press journal: Cancer Management and Research

\section{Rirong Qu (D) \\ Dehao Tu (D) \\ Wei Ping \\ Xiangning Fu}

Department of Thoracic Surgery, Tongji Hospital, Tongji Medical College, Huazhong University of Science and Technology, Wuhan, 430030, People's Republic of China
Correspondence: Xiangning Fu Department of Thoracic Surgery, Tongji Hospital, Tongji Medical College, Huazhong University of Science and Technology, Wuhan, 430030, People's Republic of China

Tel +86 I3607I50390

Fax +86 027-836652II

Email fuxn2006@aliyun.com
Background: The objective of this study was to assess the impact of the recurrent laryngeal nerve injury (RLNI) after esophagectomy on prognosis.

Methods: Retrospectively collected data from 297 patients with esophageal squamous cell carcinoma (ESCC) who underwent McKeown esophagectomy at our department from April 2014 to May 2018, were analyzed.

Results: RLNI occurred in $31.9 \%$ of the patients. Left-side RLNI occurred 2.8 times more often than right-side RLNI. Among the cases in which assessment of the vocal cords was continued, $8.4 \%$ involved permanent injury. There were no significant differences among clinicopathological data between patients with RLNI and without. Compared with patients without RLNI, patients with RNLI have longer operation time, more number of bronchoscopy suctions, longer postoperation hospital stay, and higher incidence of postoperative complications. T stage, $\mathrm{N}$ stage, RLN lymph node metastasis were independent risk factors for the prognosis, but RLNI is not independent risk factors for long-term survival.

Conclusion: RLNI is a serious complication that will affect the short-term prognosis of patients and reduce the quality of life of patients. It should be avoided as much as possible during surgery, but it may not have negative impact on the long-term survival.

Keywords: esophageal cancer, recurrent laryngeal nerve, injury, esophagectomy, prognosis

\section{Introduction}

Esophageal cancer is one of the most common malignant tumors in clinical practice. At present, it is still the eighth most common malignant tumor and the sixth malignant tumor causing death in the world. ${ }^{1,3}$ Esophageal cancer is highly invasive and has a poor prognosis, and the 5-year survival rate worldwide is only $15 \%-25 \%{ }^{2,3}$ In esophageal squamous cell carcinoma, the metastasis rate of recurrent laryngeal nerve chain lymph nodes is as high as $20 \%-40 \%{ }^{4-10}$ Therefore, dissection of bilateral recurrent laryngeal nerve chain lymph nodes is extremely important and has a positive impact on the prognosis. However, in many medical centers, ${ }^{11,12}$ dissection of bilateral recurrent laryngeal nerve chain lymph nodes is not a routine procedure for radical esophageal cancer, mainly because dissection of the recurrent laryngeal nerve chain lymph node has potential risks. Studies ${ }^{9,13,14}$ have reported that the incidence of recurrent laryngeal nerve palsy after bilateral recurrent laryngeal nerve chain lymph node dissection is as high as $60 \%$, and postoperative complications also increase obviously, especially aspiration pneumonia, which seriously affects the quality of life 
of patients, and sometimes even cause death. Although we all know that recurrent laryngeal nerve injury is one of the most common complications of recurrent laryngeal lymph node dissection, it is still unknown whether the injury will affect the long-term prognosis of patients. Therefore, this study retrospectively analyzed the incidence of recurrent laryngeal nerve injury and the postoperative follow-up data of patients with radical Mckeown esophagectomy, and explored its impact on long-term survival.

\section{Patients and Methods}

\section{Patients}

A total of 297 patients with ESCC who underwent McKeown esophagectomy at Wuhan Tongji Hospital from April 2014 to May 2018 were reviewed. These patients were diagnosed with ESCC by using an electronic gastroscope before surgery. Each patient underwent esophagography, chest and abdomen computed tomography (CT) scans, ultrasonography of the abdomen, neck, and ultrasonic gastroscopy, as well as positron emission tomography-CT (PET-CT) scans when needed.

The inclusion criteria were as follows: the patient underwent three-incision minimally invasive esophagectomy; there was no distant metastasis; except for other malignancies; no preoperative chemotherapy or radiotherapy; no combined with severe diseases of the heart, liver, lung or kidney disease, no other surgical contraindications; patients could tolerate surgical treatment.

The exclusion criteria were as follows: patients who did not undergo RLN lymph node dissection; cases that were combined with other malignant tumors; pathological results revealing other cellular components in addition to squamous cell carcinoma; patients who had hoarseness before surgery; incomplete clinicopathological data. This retrospective study was approved by the institutional review board of Tongji Medical College of Huazhong University of Science and Technology and informed written consent was obtained from all individual participants included in the study. This study was also conducted in accordance with the Declaration of Helsinki.

\section{Surgical Procedures}

All patients underwent Mckeown esophagectomy that is cervical, thoracoabdominal three-incision esophageal cancer radical surgery, and chest and abdominal cavity sections were subjected to minimally invasive surgery.
In thoracoscopic phase, double-lumen endotracheal intubation anesthesia: The patient was assumed a left lateral decubitus. The right axillary midline 5 th or 6 th intercostal $2.0-2.5 \mathrm{~cm}$ incision is a thoracoscopy hole, and the right anterior axillary line $3 \mathrm{rd}$ or 4 th intercostal $2.0-2.5 \mathrm{~cm}$ incision is an operation hole, and incision protective sleeves are placed. A two-port thoracoscopy for thoracic esophageal, RLN lymph node dissection and mediastinal lymph node dissection was used. After the thoracoscopic phase, the patient was placed in a supine position. The gastric conduit was made and abdominal lymph node dissection was performed during the laparoscopic operation. Left cervical collar middle line incisions were performed at the same time, along with cervical lymph node sampling. Last, the gastric conduit was pulled up to the left neck through the posterior mediastinum, and assisted by the stapler to enable esophagogastric anastomosis. The detailed operation of each part is described in the previous study. ${ }^{15}$

Notably, RLN lymph node dissection is performed by detecting and dissecting the entire RLN. We separate the RLN with ultrasound scissors and laparoscopic scissors, and minimize the use of electric hooks to avoid damage to the recurrent laryngeal nerve during the RLN lymph node dissection. The right RLN lymph node dissection extends from the lower edge of the brachiocephalic artery to the lower edge of thyroid, the right RLN was exposed to the level of the right subclavian artery and the surrounding LNs and fatty tissues were removed. The left extends from the upper edge of the aortic arch to the lower edge of thyroid. The 1-mm diameter nerve was separated to the root of the neck, and the surrounding LNs and fatty tissues were cleared. Since we performed preoperative evaluation of the patients' bilateral cervical and supraclavicular lymph nodes, we did not perform the supraclavicular LN dissection routinely. All specimens were pathologically diagnosed at the Tongji Hospital Department of Pathology. UICC 8th edition was used for TNM classification, and the pathological results were used for the primary tumour (T) and LN (N) scales in this study.

\section{Definition of Outcomes}

In all cases, RLNI was evaluated by fiberoptic bronchoscopy on the day of extubation. RLNI was defined as any dysmotility in the vocal cords. We routinely evaluated the patients by upper gastrointestinal fibroscopy twice a year to assess the residual oesophagus and the status of RLNI. When patients mentioned that they felt some change in their voices, such hoarseness was checked, and improvement of that hoarseness 
was defined as the patients feeling relief from the symptom, whereas permanent injury was defined as no improvement in vocal cord motility years after surgery. Patients were undetermined if the patient's vocal cord could not be assessed after discharge or if the patient's records could not be obtained. We defined aspiration as cases in which the patient began oral ingestion but, due to choking, needed some rehabilitation or special meals to promote swallowing. Anastomosis leakage was confirmed in all cases by endoscopy or by contrast radiography. Postoperative pneumonia was defined as follows: an infiltration shadow on chest radiography, a demonstrated increase in inflammation and the administration of antibiotics for pneumonia.

\section{Statistical Methods}

Data were analyzed using SPSS 23.0 software. Measured data were expressed as mean \pm standard deviation (SD) and differences between groups were analyzed by t-tests. Counted data were expressed as number or percent, and differences were analyzed using $\mathrm{X}^{2}$ or Fisher's exact tests. Overall survival (OS) was analyzed using the KaplanMeier methods and included all causes of death. The statistical significance of the survival differences was compared by the Log rank test. Univariate logistic regression analysis was carried out to analyze factors that might have affected OS, and those factors $(\mathrm{P}<0.05)$ were further analyzed using multivariate logistic regression. $\mathrm{P}<0.05$ was considered statistically significant.

\section{Results}

\section{Clinicopathological Data of Patients}

Patient and tumor characteristics are shown in Table 1. There were no significant differences among clinicopathological data between patients with RLNI and without.

\section{Perioperative Results}

Compared with patients without RLNI, patients with RNLI have longer operation time of Chest $(\mathrm{P}=0.042)$, longer average time to start liquid diet $(\mathrm{P}=0.021)$, more number of bronchoscopy suctions $(\mathrm{P}=0.032)$, longer postoperation hospital stay $(\mathrm{P}=0.047)$. In terms of postoperation complications, there was significant difference between the two groups, especially in postoperative pneumonia, need of tracheostomy, hoarse voice, but there was no significant difference in hospital mortality $(\mathrm{P}>0.05)$. In terms of lymph node dissection, patients with RNLI had more number of RLN lymph nodes dissected $(\mathrm{P}=0.034)$; there
Table I Clinicopathological Characteristics of Patients

\begin{tabular}{|c|c|c|c|c|}
\hline Variables & All Patients & RLNI (+) & RLNI (-) & $P$ value \\
\hline Total & 297 & 95 & 202 & \\
\hline Age, years & & & & 0.433 \\
\hline$<60$ & $134(45.1)$ & $46(48.4)$ & $88(43.6)$ & \\
\hline$\geq 60$ & $163(54.9)$ & $49(51.6)$ & II 4 (56.4) & \\
\hline Gender & & & & 0.628 \\
\hline Male & 207 (69.7) & 68 (7I.6) & $139(68.8)$ & \\
\hline Female & $90(30.3)$ & $27(28.4)$ & $63(3 \mid .2)$ & \\
\hline Smoking history & & & & 0.315 \\
\hline Yes & 204 (68.9) & 69 (72.6) & $135(66.8)$ & \\
\hline No & $93(31.1)$ & $26(27.4)$ & $67(33.1)$ & \\
\hline PT stage & & & & 0.514 \\
\hline TI & $30(10.1)$ & II (II.6) & $19(9.4)$ & \\
\hline T2 & $125(42.1)$ & $43(45.3)$ & $82(40.6)$ & \\
\hline T3 & 115 (38.7) & 31 (32.6) & 84 (4I.6) & \\
\hline T4 & $27(9.1)$ & $10(10.5)$ & $17(8.4)$ & \\
\hline $\mathrm{pN}$ stage & & & & 0.179 \\
\hline No & $92(30.9)$ & $26(27.4)$ & 66 (32.7) & \\
\hline NI & $110(37.0)$ & $38(40.0)$ & $72(35.6)$ & \\
\hline N2 & $65(22.0)$ & $17(17.9)$ & $48(23.8)$ & \\
\hline N3 & $30(10.1)$ & $14(14.7)$ & $16(7.9)$ & \\
\hline pTNM stage & & & & 0.753 \\
\hline IA & $78(26.3)$ & $24(25.2)$ & 54 (26.7) & \\
\hline IB & $18(6.1)$ & $4(2.2)$ & $14(6.9)$ & \\
\hline$\| A$ & $30(10.1)$ & $10(10.5)$ & $19(9.4)$ & \\
\hline IIB & $32(10.8)$ & $12(12.6)$ & $20(9.9)$ & \\
\hline$ш \mathrm{~A}$ & $73(24.6)$ & $24(25.2)$ & $49(24.3)$ & \\
\hline шB & $27(9.1)$ & II (II.6) & $16(7.9)$ & \\
\hline шC & $25(8.4)$ & $8(8.4)$ & $17(8.4)$ & \\
\hline IV & $14(4.7)$ & $2(2.1)$ & $12(5.9)$ & \\
\hline Tumor location & & & & 0.662 \\
\hline Upper & $31(10.4)$ & $10(10.5)$ & $21(10.5)$ & \\
\hline Middle & $148(49.9)$ & $49(51.6)$ & $99(49.0)$ & \\
\hline Lower & 118 (39.7) & 36 (37.9) & $92(45.5)$ & \\
\hline Adjuvant therapy & & & & 0.078 \\
\hline Yes & $98(32.9)$ & $38(40.0)$ & $60(29.7)$ & \\
\hline No & $199(67.1)$ & $57(60.0)$ & $142(70.3)$ & \\
\hline
\end{tabular}

Abbreviation: RLNI, recurrent laryngeal nerve injury.

was no significant difference between the two groups in the total number of thoracic lymph node dissected, the number of left and right recurrent laryngeal nerve lymph nodes dissected. The perioperative results are shown in Table 2 .

\section{Details of RLN Injury}

Table 3 shows that RLNI occurred in $31.9 \%$ of the patients. Among the cases in which assessment of the vocal cords was continued, $8.4 \%$ involved permanent 
Table 2 Comparison of Perioperative Between Patients with RLNI and without

\begin{tabular}{|l|c|c|c|}
\hline Variables & RLNI (+) & RLNI (-) & P value \\
\hline Operation time (min) & $185.9 \pm 25.9$ & $154 \pm 15.4$ & 0.042 \\
Blood loss (mL) & $130.3 \pm 72.7$ & $128.3 \pm 67.9$ & 0.151 \\
No. total of dissected thoracic LN & $16.15 \pm 6.7$ & $15.12 \pm 6.8$ & 0.982 \\
No. of dissected RLN LN & $6.50 \pm 4.4$ & $4.62 \pm 3.5$ & 0.034 \\
No. of dissected right RLN LN & $2.66 \pm 2.4$ & $3.62 \pm 2.4$ & 0.073 \\
No. of dissected left RLN LN & $2.87 \pm 3.1$ & $2.01 \pm 2.4$ & 0.094 \\
Average time to start liquid diet (day) & $10.23 \pm 5.2$ & $5.23 \pm 3.1$ & 0.021 \\
Number of bronchoscopy suctions & $3.67 \pm 1.7$ & $2.11 \pm 1.5$ & 0.032 \\
Hospital mortality & $2(2.2)$ & $3(1.5)$ & 0.241 \\
Postoperation hospital stay (day) & $11.5 \pm 5.4$ & $7.2 \pm 4.8$ & 0.047 \\
\hline Complications & \multicolumn{3}{|c|}{} \\
\hline Postoperative pneumonia & $34(35.8)$ & $36(17.8)$ & $<0.001$ \\
Anastomotic leakage & $15(15.8)$ & $17(8.4)$ & 0.012 \\
Need of tracheostomy & $5(5.3)$ & $4(1.9)$ & $<0.001$ \\
Aspiration & $18(18.9)$ & $20(9.9)$ & 0.015 \\
Hoarse voice & $35(36.8)$ & $28(13.7)$ & $<0.001$ \\
\hline
\end{tabular}

Abbreviations: RLNI, recurrent laryngeal nerve injury; RLN LN, recurrent laryngeal nerve injury lymph node.

Table 3 Details of RLN Injury

\begin{tabular}{|l|c|}
\hline RLN Injury & Number (\%) \\
\hline Non-injury & $202(68.1)$ \\
RLN injury & $95(31.9)$ \\
Right & $21(22.1)$ \\
Left & $59(62.1)$ \\
Bilateral & $15(15.8)$ \\
\hline Type of RLN injury & \\
\hline Reduced activity on one side & $38(40.0)$ \\
Reduced activity on two sides & $27(28.4)$ \\
Paralysis of one side & $24(25.3)$ \\
Paralysis of two sides & $6(6.3)$ \\
\hline Temporary or permanent injury & $75(78.9)$ \\
\hline Temporary & $8(8.4)$ \\
Permanent & $12(12.7)$ \\
\hline Undetermined
\end{tabular}

Abbreviation: RLN, recurrent laryngeal nerve.

injury. Left-side RLNI occurred 2.8 times more often than right-side RLNI. According to type of RLN injury, among patients with RLNI, $68.4 \%$ of patients were with reduced activity on vocal cords (Figure 1). Through our long-term follow-up, the mean and median times of improvement of hoarseness were 125.5 and 97.5 days, respectively, ranging from 6 to 455 days, among patients with temporary injury.

\section{Correlation Between RLNI and OS}

The relationships between RLNI and OS of patients with esophageal cancer were analyzed by Log rank test. The results showed that there was no significant difference between patients with RLNI and without (Figure 2).

\section{Univariate and Multivariate Analysis of Prognostic Factors Influencing the Survival of Patients}

Univariate regression analysis showed that $\mathrm{T}$ stage, $\mathrm{N}$ stage, tumor location, RLNI and RLN LN metastasis were risk factors for the prognosis of patients with esophageal cancer (Table 4). The dependent variable was used for the prognosis of patients with the above risk factors. The differences that were statistically significant in univariate analysis were used as independent variables. The Cox regression model was used for multivariate analysis. The results showed that $\mathrm{T}$ stage, $\mathrm{N}$ stage, RLN lymph node metastasis were independent risk factors for the prognosis, but RLNI is not independent risk factors for long-term survival (Table 5).

\section{Follow-Up and Survival}

Patients who were discharged smoothly after surgery were followed up, either in the outpatient clinic or by telephone every three months during the first year, including how the patients with hoarseness recovered after surgery, postoperative feeding, and recent chest and abdominal CT examinations. Upper gastrointestinal fibroscopy was performed twice a year. OS was calculated from the date of operation to the occurrence of death or to the last known date of follow-up.

The average follow-up time was 33 months (9-58 months). During the follow-up, we found that most patients still died of tumor recurrence and metastasis, and only five patients died of aspiration caused by permanent recurrent laryngeal nerve injury. There was no significant difference between patients with RLNI and without in OS.

\section{Discussion}

In recent years, with the application of neoadjuvant chemoradiotherapy + surgery + adjuvant therapy and other treatment modes, the survival rate of esophageal cancer patients has improved, but 5-year overall survival rate is still less than $35 \%$ in the world. Several studies ${ }^{16,17}$ have shown that the poor prognosis of esophageal cancer is mainly related to the 


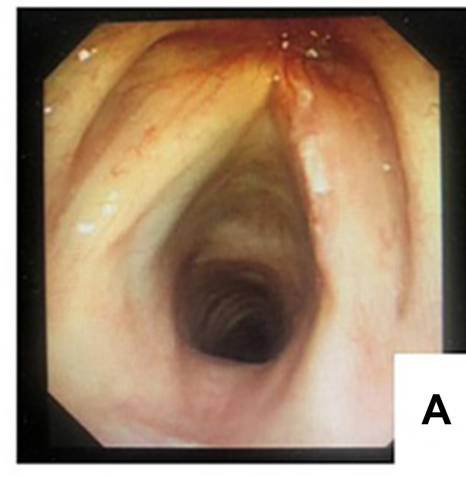

A.Normal vocal cord activity

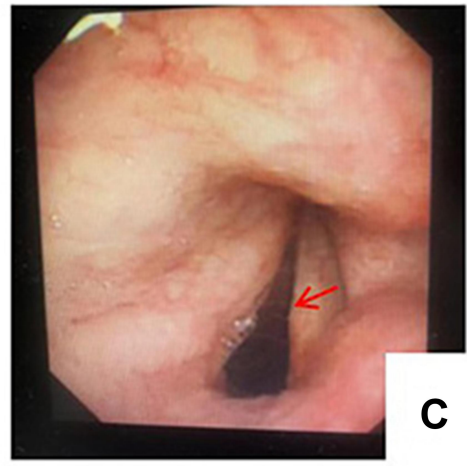

C.Abnormal right-side vocal cord activity

Figure I The vocal cords after recurrent laryngeal nerve injury.

recurrence and metastasis of postoperative lymph nodes, especially the recurrence and metastasis of bilateral recurrent laryngeal nerve chain lymph nodes. Therefore, in the radical operation of esophageal cancer, the dissection of bilateral recurrent laryngeal nerve chain lymph nodes is extremely important. Lymph node dissection of bilateral recurrent laryngeal nerve chain can undoubtedly improve the staging accuracy of patients with esophageal cancer and improve the prognosis of patients to a certain extent, but it also increases the incidence of recurrent laryngeal nerve injury. ${ }^{18-20}$

When performing bilateral recurrent laryngeal lymph node dissection, the incidence of recurrent laryngeal nerve palsy after surgery ranges from $13 \%$ to $80 \%{ }^{9,21-23}$ Compared to the right recurrent laryngeal nerve, the incidence of the left recurrent laryngeal nerve palsy is higher. In this study, the rate of RLNI was $31.9 \%$ in all patients, left-side RLNI occurred 2.8 times more often than right-side RLNI. Regarding the rate of the RLNI, our center has a higher incidence than previous studies. ${ }^{19,24,25}$ There are some reasons: First, our center uses fiberoptic bronchoscopy to observe vocal cord activity to determine whether the recurrent laryngeal nerve is damaged. On the one hand, the vocal cord activity is checked, and on the

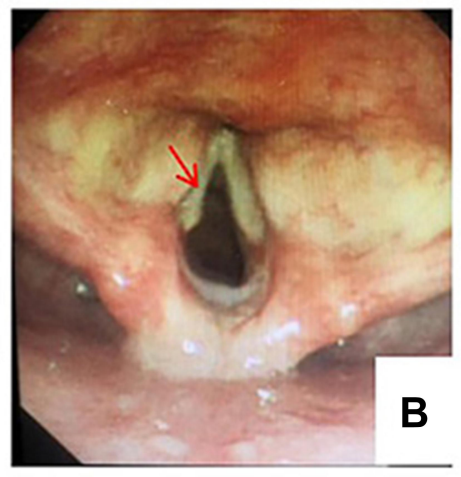

B.Abnormal left-side vocal cord activity

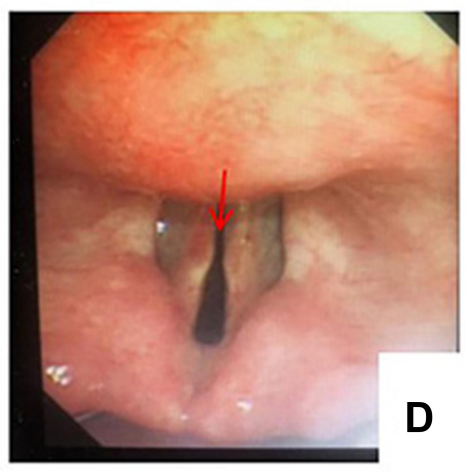

D.Abnormal bilateral vocal cord activity

other hand, the patient is suctioned to prevent pneumonia. In other centers, RLNI was either diagnosed or previously suspected by clinical symptoms. Second, the stretching, bending, and thermal damage for the nerve might be the reason for it during LN dissection. Third, after the tracheal intubation is removed, we examined the vocal cords by the fiberoptic bronchoscopy routinely. In some patients, the recurrent laryngeal nerve injury is caused by tracheal intubation probably due to the nerve's ischemic change, which may be significantly improved after one or two days.

In this study, we found that patients with RLNI have a longer operation time of chest and higher incidence of postoperative complications, especially respiratory complications. Our results could be explained from the following points: First, the longer the operation time, which means that the surgical trauma is greater, and it is more likely that it will take longer to clean the recurrent laryngeal nerve chain lymph nodes, resulting in a higher probability of RLNI; Second, the RLNI can cause temporary or permanent injury of vocal cord, which could cause serious persistent hoarseness, weak cough and expectoration leading to postoperative pneumonia, tracheal intubation or tracheotomy is needed in 


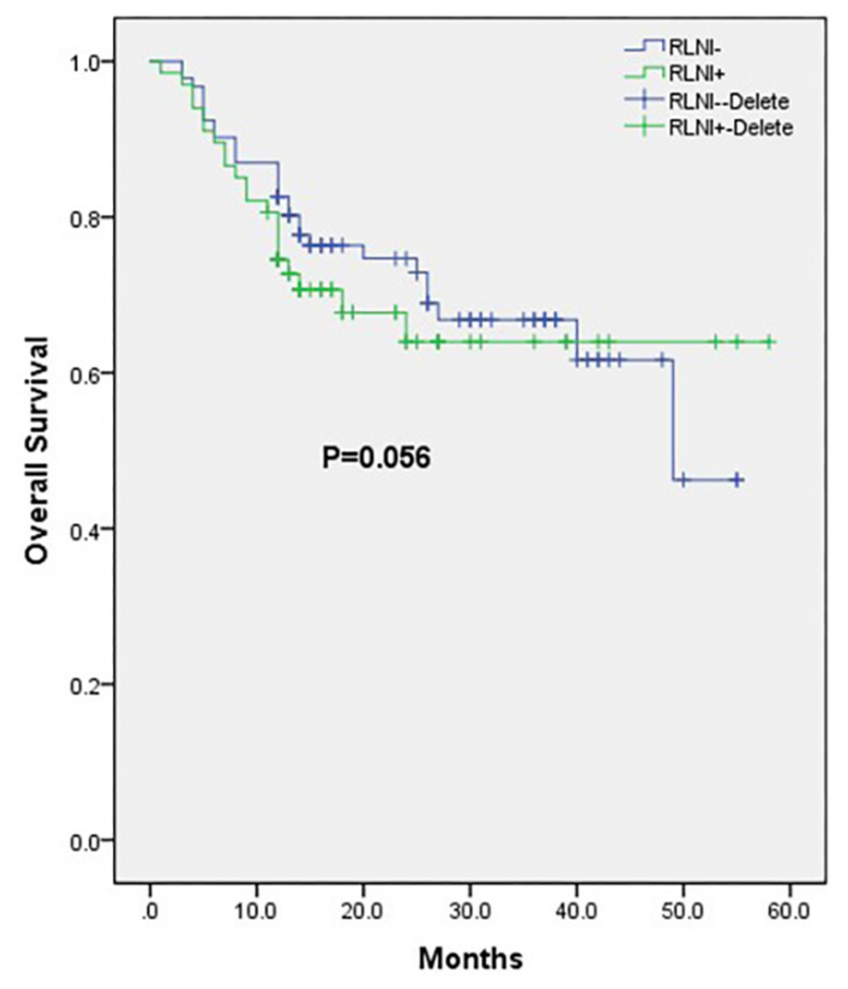

Figure 2 The relationships between RLNI and OS of patients with esophageal cancer were analyzed by Log rank test. The results showed that were no significant difference between patients with RLNI and without ( $P>0.05)$.

severe cases, and it will affect patients' postoperative rehabilitation and quality of life, and is even life-threatening. Therefore, we do believe that RLNI will adversely affect the short-term prognosis of patients and should be avoided as much as possible during the operation.

There is no consensus on whether postoperative complications of esophageal cancer will affect the overall survival of patients. Most scholars ${ }^{26,27}$ reported that postoperative complications will have a negative impact on the long-term survival. However, some scholars ${ }^{28,29}$

Table 4 Univariate Analysis of Prognostic Factors Influencing the Survival of Patients

\begin{tabular}{|l|c|c|c|}
\hline Prognostic Factors & HR & $\mathbf{9 5 \%} \mathbf{~ C l}$ & P value \\
\hline Age & 1.287 & $0.710-2.231$ & 0.123 \\
Sex & 1.053 & $0.845-1.320$ & 0.542 \\
T stage & 0.475 & $0.325-0.710$ & $<0.001$ \\
N stage & 0.384 & $0.298-0.645$ & $<0.001$ \\
Tumor location & 1.312 & $1.104-1.623$ & $0.43 \mathrm{I}$ \\
RLN LN metastasis & 3.453 & $2.432-4.656$ & $<0.00 \mathrm{I}$ \\
RLN injury & 1.412 & $0.892-1.543$ & 0.056 \\
\hline
\end{tabular}

Abbreviations: RLN, recurrent laryngeal nerve; $\mathrm{HR}$, hazard ratio; $\mathrm{Cl}$, confidence interval; LN, lymph node.
Table 5 Multivariate Analysis of Prognostic Factors Influencing the Survival of Patients

\begin{tabular}{|l|l|l|l|}
\hline Prognostic Factors & HR & $\mathbf{9 5 \%} \mathbf{C l}$ & P value \\
\hline T stage: TI+T2/T3+T4 & 1.489 & $1.232-1.908$ & $<0.001$ \\
N stage:N0+NI/N2+N3 & 1.235 & $0.987-1.876$ & $<0.001$ \\
Location of tumor: upper/others & 1.321 & $0.965-1.589$ & 0.123 \\
RLN LN metastasis (yes/no) & 3.321 & $2.412-4.578$ & $<0.00 \mathrm{I}$ \\
\hline
\end{tabular}

Abbreviations: RLN, recurrent laryngeal nerve; $\mathrm{HR}$, hazard ratio; $\mathrm{Cl}$, Confidence interval; LN, lymph node.

found that postoperative complications will not affect long-term prognosis. In terms of RLNI, researchers ${ }^{19,20,22}$ have found that it can adversely affect the short-term prognosis of the patient, but it is still unknown whether it will affect the long-term prognosis. To our best knowledge, this study is one of the few studies on the long-term prognosis of patients with RLNI after esophagectomy. Using Cox Multivariate regression analysis, we found that $\mathrm{T}$ stage, $\mathrm{N}$ stage, and RLN LN metastasis were independent prognostic factors of OS, but RLNI did not affect OS (HR=1.412, 95\% CI: $0.892-1.543, \mathrm{P}=0.056$ ). Through our postoperative follow-up, we also found that patients with hoarseness generally recover within 3-6 months after surgery, and it is extremely rare to have water cough and eating difficulties, which is consistent with the Baba $\mathrm{M}$ et al. ${ }^{25}$ Therefore, we believe that major of the recurrent laryngeal nerve injury is temporary, most patients can recover through functional exercise and nutritional neurotherapy after surgery, so there is no significant effect on the overall survival of patients.

However, our study had some limitations and shortcomings. The first and a major limitation of this study is its retrospective nature, thus, the selected bias definitely existed. Second, there were no subgroup analyses between different clinical stage with RLNI in the study. However, there was no significant difference in general clinical data between the two groups of patients in the study, which may compensate for this deficiency. Last, the sample size of this study in the single institution is small, and it needs to be confirmed by prospective, multi-institutional and large sample studies in the future.

\section{Conclusions}

RLNI is a serious complication that will affect the shortterm prognosis of patients and reduce the quality of patient's life. It should be avoided as much as possible 
during surgery, but it may not have negative impact on the long-term survival.

\section{Disclosure}

None of the authors have any potential conflicts of interest.

\section{References}

1. Bray F, Ferlay J, Siegel RL, et al. Global cancer statistics 2018: GLOBOCAN estimates of incidence and mortality worldwide for 36 cancers in 185 countries. CA Cancer J Clin. 2018;68 (6):394-424. doi:10.3322/caac. 21492

2. Straatman J, Joosten PJ, Terwee CB, et al. Systematic review of patient reported outcome measures in the surgical treatment of patients with esophageal cancer. Dis Esophagus. 2016;29 (7):760-772. doi:10.1111/dote.12405

3. Pennathur A, Gibson MK, Jobe BA, et al. Oesophageal carcinoma. Lancet. 2013;381(9864):400-412. doi:10.1016/S0140-6736(12) 60643-6

4. Ye K, Xu JH, Sun YF, et al. Characteristics and clinical significance of lymph node metastases near the recurrent laryngeal nerve from thoracic esophageal carcinoma. Genet Mol Res. 2014;13 (3):6411-6419. doi:10.4238/2014.August.25.4

5. Li B, Chen H, Xiang J, et al. Pattern of lymphatic spread in thoracic esophageal squamous cell carcinoma: a single-institution experience. $J$ Thorac Cardiovasc Surg. 2012;144(4):778-785. doi:10.1016/j. jtcvs.2012.07.002

6. Sato F, Shimada Y, Li Z, et al. Paratracheal lymph node metastasis is associated with cervical lymph node metastasis in patients with thoracic esophageal squamous cell carcinoma. Ann Surg Oncol. 2002;9(1):65-70. doi:10.1245/aso.2002.9.1.65

7. Wu J, Chen QX, Zhou XM, et al. Does recurrent laryngeal nerve lymph node metastasis really affect the prognosis in node-positive patients with squamous cell carcinoma of the middle thoracic esophagus? BMC Surg. 2014;14:43. doi:10.1186/1471-2482-14-43

8. Li H, Yang S, Zhang Y, et al. Thoracic recurrent laryngeal lymph node metastases predict cervical node metastases and benefit from three-field dissection in selected patients with thoracic esophageal squamous cell carcinoma. J Surg Oncol. 2012;105(6):548-552. doi:10.1002/jso. 22148

9. Taniyama Y, Miyata G, Kamei T, et al. Complications following recurrent laryngeal nerve lymph node dissection in oesophageal cancer surgery. Interact Cardiovasc Thorac Surg. 2015;20(1):41-46. doi:10.1093/icvts/ivu336

10. Tan Z, Ma G, Zhao J, et al. Impact of thoracic recurrent laryngeal node dissection: 508 patients with tri-incisional esophagectomy. J Gastrointest Surg. 2014;18(1):187-193. doi:10.1007/s11605-013-2411-2

11. Law S, Wong J. Lymph node dissection in surgical treatment of esophageal neoplasms. Surg Oncol Clin N Am. 2007;16(1):115-131. doi:10.1016/j.soc.2006.10.008

12. Tong D, Law S. Extended lymphadenectomy in esophageal cancer is crucial. World J Surg. 2013;37(8):1751-1756. doi:10.1007/s00268013-2068-8

13. Sato Y, Kosugi S-I, Aizawa N, et al. Risk factors and clinical outcomes of recurrent laryngeal nerve paralysis after esophagectomy for thoracic esophageal carcinoma. World J Surg. 2016;40(1):129-136. doi:10.1007/s00268-015-3261-8

14. Pertl L, Zacherl J, Mancusi G, et al. High risk of unilateral recurrent laryngeal nerve paralysis after esophagectomy using cervical anastomosis. Eur Arch Otorhinolaryngol. 2011;268(11):1605-1610. doi:10.1007/s00405-011-1679-7
15. Wang Q, Ping W, Cai Y, et al. Modified McKeown procedure with uniportal thoracoscope for upper or middle esophageal cancer: initial experience and preliminary results. $J$ Thorac Dis. 2019;11 (11):4501-4506. doi:10.21037/jtd.2019.11.07

16. Nakagawa S, Kanda T, Kosugi S, et al. Recurrence pattern of squamous cell carcinoma of the thoracic esophagus after extended radical esophagectomy with three-field lymphadenectomy. J Am Coll Surg. 2004;198(2):205-211. doi:10.1016/j.jamcollsurg.2003.10.005

17. Li CL, Zhang FL, Wang YD, et al. Characteristics of recurrence after radical esophagectomy with two-field lymph node dissection for thoracic esophageal cancer. Oncol Lett. 2013;5(1):355-359. doi:10.3892/ol.2012.946

18. Ma L, Xiang J, Zhang Y, et al. Characteristics and clinical significance of recurrent laryngeal nerve lymph node metastasis in esophageal squamous cell carcinoma. J BUON. 2017;22(6):1533-1539.

19. Gockel I, Kneist W, Keilmann A, et al. Recurrent laryngeal nerve paralysis (RLNP) following esophagectomy for carcinoma. Eur J Surg Oncol. 2005;31(3):277-281. doi:10.1016/j.ejso.2004.10.007

20. Booka E, Takeuchi H, et al. The impact of postoperative complications on survivals after esophagectomy for esophageal cancer. Medicine. 2015;94(33):e1369. doi:10.1097/MD.0000000000001369

21. Koyanagi K, Igaki H, Iwabu J, et al. Recurrent laryngeal nerve paralysis after esophagectomy: respiratory complications and role of nerve reconstruction. Tohoku $J$ Exp Med. 2015;237(1):1-8. doi:10.1620/tjem.237.1

22. Nishimaki T, Suzuki T, Suzuki S, et al. Outcomes of extended radical esophagectomy for thoracic esophageal cancer. J Am Coll Surg. 1998;186(3):306-312. doi:10.1016/s1072-7515(98)00013-1

23. Liu Y, Zou ZQ, Xiao J, et al. A nomogram prediction model for recurrent laryngeal nerve lymph node metastasis in thoracic oesophageal squamous cell carcinoma. $J$ Thorac Dis. 2019;11(7):2868-2877. doi: $10.21037 /$ jtd.2019.06.46

24. Hulscher JB, van Sandick JW, et al. Vocal cord paralysis after subtotal oesophagectomy. $B r \quad J \quad$ Surg. 1999;86(12):1583-1587. doi:10.1046/j.1365-2168.1999.01333.x

25. Baba M, Natsugoe S, Shimada M, et al. Does hoarseness of voice from recurrent nerve paralysis after esophagectomy for carcinoma influence patient quality of life? J Am Coll Surg. 1999;188 (3):231-236. doi:10.1016/s1072-7515(98)00295-6

26. Rizk NP, Bach PB, Schrag D, et al. The impact of complications on outcomes after resection for esophageal and gastroesophageal junction carcinoma. J Am Coll Surg. 2004;198(1):42-50. doi:10.1016/j. jamcollsurg.2003.08.007

27. Lerut T, Moons J, Coosemans W, et al. Postoperative complications after transthoracic esophagectomy for cancer of the esophagus and gastroesophageal junction are correlated with early cancer recurrence: role of systematic grading of complications using the modified Clavien classification. Ann Surg. 2009;250(5):798-807. doi:10.1097/ SLA.0b013e3181bdd5a8

28. Lindner K, Fritz M, Haane C, et al. Postoperative complications do not affect long-term outcome in esophageal cancer patients. World J Surg. 2014;38(10):2652-2661. doi:10.1007/s00268-014-2590-3

29. D'Annoville T, D'Journo XB, Trousse D, et al. Respiratory complications after oesophagectomy for cancer do not affect disease-free survival. Eur J Cardiothorac Surg. 2012;41(5):e66-e73. doi:10.1093/ ejcts/ezs080 


\section{Publish your work in this journal}

Cancer Management and Research is an international, peer-reviewed open access journal focusing on cancer research and the optimal use of preventative and integrated treatment interventions to achieve improved outcomes, enhanced survival and quality of life for the cancer patient.

The manuscript management system is completely online and includes a very quick and fair peer-review system, which is all easy to use. Visit http://www.dovepress.com/testimonials.php to read real quotes from published authors.

Submit your manuscript here: https://www.dovepress.com/cancer-management-and-research-journal 